\title{
Factorization Method in Oscillator with the Aharonov-Casher System
}

\author{
J. Sadeghi, ${ }^{1}$ Jalil Naji, ${ }^{2,3}$ and Behnam Pourhassan ${ }^{4,5}$ \\ ${ }^{1}$ Department of Physics, Sciences Faculty, Azad University, Ayatollah Amoli, P.O. Box 678, Amol, Iran \\ ${ }^{2}$ Physics Department, Ilam University, P.O. Box 69315-516, Ilam, Iran \\ ${ }^{3}$ Department of Physics, Ilam Branch, Islamic Azad University, Ilam, Iran \\ ${ }^{4}$ Department of Physics, Damghan University, Damghan, Iran \\ ${ }^{5}$ Young Researchers Club, Islamic Azad University, Ayatollah Amoli Branch, Amol, Iran \\ Correspondence should be addressed to Jalil Naji; naji.jalil2020@gmail.com
}

Received 23 March 2014; Revised 3 April 2014; Accepted 3 April 2014; Published 22 April 2014

Academic Editor: Shi-Hai Dong

Copyright (c) 2014 J. Sadeghi et al. This is an open access article distributed under the Creative Commons Attribution License, which permits unrestricted use, distribution, and reproduction in any medium, provided the original work is properly cited.

We review the oscillator with Aharonov-Casher system and study some mathematical foundation about factorization method. The factorization method helps us to obtain the energy spectrum and general wave function for the corresponding system in some spin condition. The factorization method leads us to obtain the raising and lowering operators for the Aharonov-Casher system. The corresponding operators give us the generators of the algebra.

\section{Introduction}

As we know, the relativistic quantum dynamics of a natural particle which describes the relativistic Aharonov-Casher system [1] is given by introducing minimal coupling into the Dirac equation $[2,3]$ in Cartesian coordinates which is given by

$$
i \gamma^{\mu} \partial_{\mu} \longrightarrow i \gamma^{\mu} \partial_{\mu}+\frac{\mu}{2} \sum^{\mu \nu} F_{\mu \nu}(x)
$$

where we consider the natural units $\hbar=c=1$. Also, $\mu$ corresponds to the magnetic dipole moment of natural particle, and $F_{\mu \nu}(x)$ corresponds to the electromagnetic tensor, which are defined by $F_{0 i}=-F_{i 0}=E_{i}$ and $F_{i j}=-F_{j i}=-\epsilon_{i j k} B^{k}$ and $\sum^{a b}=(i / 2)\left[\gamma^{a}, \gamma^{b}\right]$. The $\gamma^{a}$ matrices correspond to the Dirac matrices in Minkowski space-time [4]:

$$
\begin{gathered}
\gamma^{0}=\widehat{\beta}=\left(\begin{array}{cc}
1 & 0 \\
0 & -1
\end{array}\right), \quad \gamma^{i}=\widehat{\beta} \widehat{\alpha}^{i}=\left(\begin{array}{cc}
0 & \sigma^{i} \\
-\sigma^{i} & 0
\end{array}\right), \\
\sum^{i}=\left(\begin{array}{cc}
\sigma^{i} & 0 \\
0 & \sigma^{i}
\end{array}\right),
\end{gathered}
$$

with $\gamma^{a} \gamma^{b}+\gamma^{b} \gamma^{a}=-2 \eta^{a b}$, with $\vec{\sum}$ being the spin vector and $\sigma^{i}$ being Pauli matrices. The tensor $\eta^{a b}=\operatorname{diag}(-+++)$ is the Minkowski tenor. Moreover, by introducing the coupling that describes the Dirac oscillator $\vec{P} \rightarrow \vec{P}-i m \omega \rho \widehat{\beta} \hat{\rho}$ into the nonminimal coupling (1), we can see that the whole system is cylindrically symmetric. So, we can work with curvilinear coordinates $x=\rho \cos \varphi$ and $y=\rho \sin \varphi$. Therefore, we write the line element of the Minkowski space-time in the following form:

$$
d s^{2}=-d t^{2}+d \rho^{2}+\rho^{2} d \varphi^{2}+d z^{2} .
$$

Here, we note that, in curvilinear coordinate (both flat and curved space-time background), the relativistic quantum dynamics of a neutral particle with a permanent magnetic dipole moment interacting with the external field is not described by the Dirac equation with the introduction of nonminimal coupling (1) anymore. Based on the spinor theory in curved space-time, nonminimal coupling (1) plus the coupling describing the Dirac oscillator becomes

$$
\begin{aligned}
i \gamma^{\mu} \nabla_{\mu} & \longrightarrow i \gamma^{\mu} \partial_{\mu}+i \gamma^{\mu} \Gamma_{\mu}(x)+i \gamma^{\mu} m \omega \rho \gamma^{0}+\delta_{\mu}^{\rho} \\
& +\frac{\mu}{2} \sum^{\mu \nu} F_{\mu \nu}(x),
\end{aligned}
$$


where $\nabla_{\mu}+\partial_{\mu}+\Gamma_{\mu}(x)$ corresponds to the components of the covariant derivative of a spinor, with $\Gamma_{\mu}=(i / 4) \omega_{\mu a b}(x) \sum^{a b}$ being the spinnorial connection $[5,6]$, and $\sum^{a b}=$ $(1 / 2)\left[\gamma^{a}, \gamma^{b}\right]$. In the spinor theory in curved space-time, the $\gamma^{a}$ matrices are defined in the local reference frame of the observers and are identical to the Dirac matrices defined in Minkowski space-time (3). In this notation, the indices $(a, b, c=0,1,2,3)$ indicate the local reference frame, while the indices $(\mu, \nu)$ indicate the space-time indices. Thus, the $\gamma^{\mu}$ matrices given by (4) are related to the $\gamma^{a}$ via $\gamma^{\mu}=e_{a}^{\mu}(x) \gamma^{a}$, where components $e_{a}^{\mu}(x)$ are called tetrades and give rise to the local reference frame of the observers. The tetrades satisfy the following equation $[5,6]$ :

$$
g_{\mu \nu}(x)=e_{\mu}^{a}(x) e_{\nu}^{b}(x) \eta_{a b} .
$$

The tetrades also have an inverse defined as $d x^{\mu}=e_{a}^{\mu}(x) \theta^{a}$, where

$$
\begin{aligned}
& e_{\mu}^{a}(x) e_{b}^{\mu}(x)=\delta_{b}^{a}, \\
& e_{a}^{\mu}(x) e_{\nu}^{a}(x)=\delta_{\nu}^{\mu} .
\end{aligned}
$$

Furthermore, the components of the spinnorial connection can be obtained by solving the Cartan structure [6] in the absence of torsion:

$$
d \widehat{\theta}^{a}+\omega_{b}^{a} \widehat{\theta}^{b}=0
$$

where $\omega_{b}^{a}=\omega_{\mu_{b}}^{a}(x) d x^{\mu}$ and $\omega_{\mu_{b}}^{a}(x)$ are called connection 1 -form. For instance, we can choose the tetrades for line element (3) being

$$
\hat{\theta}^{\theta}=d t, \quad \hat{\theta}^{1}=d \rho, \quad \widehat{\theta}^{2}=\rho d \varphi, \quad \hat{\theta}^{3}=d z .
$$

By solving the Cartan structure equations in the absence of torsion, we can obtain $\omega_{\varphi_{2}}^{1}(x)=-\omega_{\varphi_{1}}^{2}(x)=-1$ and $\gamma^{\mu} \Gamma_{\mu}(x)=$ $\gamma^{1} / 2 \rho$. Hence, the Dirac equation describing the interaction between the Dirac oscillator and Aharonov-Casher system is

$$
\begin{aligned}
m \Psi= & i \gamma^{0} \frac{\partial \Psi}{\partial t}+i \gamma^{1}\left[\frac{\partial}{\partial \rho}+\frac{1}{2 \rho}+m \omega \rho \gamma^{0}\right] \Psi+i \frac{\gamma^{2}}{\eta \rho} \frac{\partial \Psi}{\partial \varphi} \\
& +i \gamma^{3} \frac{\partial \Psi}{\partial z}+i \mu \vec{\alpha} \cdot \vec{E} \Psi-\mu \vec{\sum} \vec{B} \Psi .
\end{aligned}
$$

Here, we consider radial electric field as $\vec{E}=(\lambda / \rho) \widehat{\rho}$ and also consider the magnetic dipole moment parallel to the $z$-axis. In that case we can rewrite the Dirac equation as

$$
\begin{aligned}
i \frac{\partial \Psi}{\partial t}= & m \widehat{\beta} \Psi-i \widehat{\alpha}^{1}\left[\frac{\partial}{\partial \rho}+\frac{1}{2 \rho}+m \omega \rho \widehat{\beta}\right] \Psi-i \frac{\widehat{\alpha}^{2}}{\rho} \frac{\partial \Psi}{\partial \varphi} \\
& -i \widehat{\alpha}^{3} \frac{\partial \Psi}{\partial z}-i \frac{\Phi_{A C}}{2 \pi \rho} \widehat{\beta} \cdot \widehat{\alpha}^{1} \Psi,
\end{aligned}
$$

where $\Phi_{A C}= \pm 2 \pi(\mu \lambda / \hbar c)=(1 / \hbar c) \oint(\vec{M} \times \vec{E})_{\nu} d x^{\nu}$ is Aharonov-Casher geometric phase [1]. In order to solve (10) one can write $\Psi$ in terms of two components of spinors as

$$
\Psi=e^{-i E t}\left(\begin{array}{l}
\phi \\
\psi
\end{array}\right)
$$

where $\phi$ and $\psi$ are spinors of two components. We substitute (11) in (10) to obtain two coupled equations for $\phi$ and $\psi$. Now, we are going to write the first coupled equation which is given by

$$
\begin{aligned}
(\varepsilon-m) \phi= & -i \delta^{1}\left[\frac{\partial}{\partial \rho}+\frac{1}{2 \rho}+\frac{\Phi_{A C}}{2 \pi \rho}-m \omega \rho\right] \psi-i \frac{\delta^{2}}{\rho} \frac{\partial \psi}{\partial \varphi} \\
& -i \delta^{3} \frac{\partial \psi}{\partial z}
\end{aligned}
$$

and the second coupled equation is

$$
\begin{aligned}
(\varepsilon+m) \psi= & -i \delta^{1}\left[\frac{\partial}{\partial \rho}+\frac{1}{2 \rho}-\frac{\Phi_{A C}}{2 \pi \rho}+m \omega \rho\right] \phi-i \frac{\delta^{2}}{\rho} \frac{\partial \phi}{\partial \varphi} \\
& -i \delta^{3} \frac{\partial \phi}{\partial z} .
\end{aligned}
$$

By using (12) and (13) one can obtain the following second order differential equation:

$$
\begin{aligned}
\left(\varepsilon^{2}-m^{2}\right) \phi= & -\frac{\partial^{2} \phi}{\partial \rho^{2}}-\frac{1}{\rho} \frac{\partial \phi}{\partial \rho}-\frac{1}{\rho^{2}} \frac{\partial^{2} \phi}{\partial \varphi^{2}}-\frac{\partial^{2} \phi}{\partial z^{2}} \\
& +i \frac{\delta^{3}}{\rho^{2}} \frac{\partial \phi}{\partial \varphi}+\frac{1}{4 \rho^{2}} \phi+2 i \delta^{3} m \omega \frac{\partial \phi}{\partial \varphi}-m \omega \phi \\
& -2 i \delta^{3} \frac{\Phi_{A C}}{2 \pi \rho^{2}} \frac{\partial \phi}{\partial \varphi}-\frac{\Phi_{A C}}{2 \pi \rho^{2}} \phi+m^{2} \omega^{2} \rho^{2} \phi \\
& -2 m \omega \frac{\Phi_{A C}}{2 \pi} \phi+\left(\frac{\Phi_{A C}}{2 \pi}\right)^{2} \frac{\phi}{\rho^{2}} .
\end{aligned}
$$

Here, $\phi$ is eigenfunction of the Pauli matrix $\delta^{3}$ and total angular momentum $\widehat{J}_{z}=-i \partial_{\phi}$, and the $z$-component of the momentum $\widehat{p}_{z}=-i \partial_{z}$ commutes with the Hamiltonian of (14). In that case, we can write the solution of (14) in terms of the eigenvalues of the operators $\widehat{p}_{z}=-i \partial_{z}$ and $\widehat{J}_{z}=-i \partial_{\phi}$,

$$
\phi_{s}(\rho, \varphi, z)=A e^{i(\ell+(1 / 2)) \varphi} e^{i k z} R_{z}(\varphi) .
$$

$s= \pm 1$ give $\delta^{3} \phi_{0}=s \phi_{0}$, where $\phi_{0}=\left(\phi_{+}, \phi_{-}\right)^{T}$, with $\ell=0, \pm 1, \pm 2, \ldots, k$ being constant and $A$ being normalization factor. Thus, substituting the solution (15) into the second order differential equation (14), we obtain the following radial equation:

$$
R_{s}^{\prime \prime}(\rho)+\frac{1}{\rho} R_{s}^{\prime}(\rho)+\left(\beta_{s}-\frac{\xi_{s}^{2}}{\rho^{2}}-m^{2} \omega^{2} \rho^{2}\right) R_{s}(\rho)=0,
$$

where

$$
\begin{gathered}
\xi_{s}=\ell+\frac{1}{2}(1-s)+s \frac{\Phi_{A C}}{2 \pi}, \\
\beta_{s}=\varepsilon^{2}-m^{2}-k^{2}+2 m \omega\left(1+s \xi_{s}\right) .
\end{gathered}
$$


In order to solve (16) we change variables given by $x=m \omega \rho^{2}$ and obtain

$$
x R_{s}^{\prime \prime}(x)+R_{s}^{\prime}(x)+\left(\frac{\beta_{s}}{4 m \omega}-\frac{\xi_{s}^{2}}{4 x}-\frac{x}{4}\right) R_{s}(x)=0 .
$$

Again, we choose the change of variable as follows:

$$
R_{s}(x)=F(x) L_{n, m^{\prime}}^{\alpha, \beta}(x),
$$

so, (18) changes as follows:

$$
\begin{aligned}
& x L_{n, m}^{\prime \prime(\alpha, \beta)}(x)+\left(2 x \frac{F^{\prime}(x)}{F(x)}+1\right) L_{n, m}^{\prime(\alpha, \beta)}(x) \\
& +\left[x \frac{F^{\prime \prime}(x)}{F(x)}+\frac{F^{\prime}(x)}{F(x)}+\frac{\beta_{s}}{4 m \omega}-\frac{\xi_{s}^{2}}{4 x}-\frac{x}{4}\right] L_{n, m}^{(\alpha, \beta)}(x)=0 .
\end{aligned}
$$

In order to obtain $R_{s}(x)$ in (19), we have to compare (20) with known polynomial. For this reason, first we introduce the Laguerre polynomial which is corresponding to (20). So, in this paper first we will try to review some mathematical foundation about factorization method [7]. In Section 3 we take advantage of factorization method and obtain the energy spectrum and general wave function for the corresponding system. Also, we show that the corresponding equation can be factorized first with respect to $m^{\prime}$ and then with respect to $n$ and $m^{\prime}$. These lead us to obtain the raising and lowering operators. Note that the shape invariant equation (27) can be written as the raising and lowering relations for the Aharonov-Casher system. These operators will be generators algebra.

\section{Mathematical Foundation}

Using the factorization approach, we compute the energy spectrum $\varepsilon$ and also bound states $\Psi$ through the comparison of the differential equation given in (18) with associated Laguerre differential equation in an appropriate manner. We also factorize the second order differential equations into new sets of operators $A^{-}, A^{+}$and shape invariant form, which are the first order differential equations. This process is called factorization method. Before anything else we will try to explain the associated Laguerre differential equation $L_{n, m^{\prime}}^{(\alpha, \beta)}(x)$ in factorization method point of view. To start, we need to recall that, for the real parameters $\alpha>-1$ and $\beta>0$, the associated Laguerre differential equation corresponding to $L_{n, m^{\prime}}^{(\alpha, \beta)}(x)$ in the interval $x \in(0, \infty)$ is introduced as $[8,9]$

$$
\begin{aligned}
x L_{n, m^{\prime}}^{\prime \prime(\alpha, \beta)}(x) & +(1+\alpha-\beta x) L_{n, m^{\prime}}^{\prime(\alpha, \beta)}(x) \\
& +\left[\left(n-\frac{m^{\prime}}{2}\right) \beta-\frac{m^{\prime}}{2 x}\left(\alpha+\frac{m^{\prime}}{2}\right)\right] L_{n, m^{\prime}}^{(\alpha, \beta)}(x)=0 .
\end{aligned}
$$

Here, the indices $n$ and $m^{\prime}$ are nonnegative integers for $0 \leq$ $m^{\prime}<n$. The associated Laguerre function, $L_{n, m^{\prime}}^{(\alpha, \beta)}(x)$ as a solution of the differential (21), has the following Rodrigues representation:

$$
L_{n, m}^{(\alpha, \beta)}(x)=\frac{\alpha_{n, m^{\prime}}(\alpha, \beta)}{x^{\alpha+\left(m^{\prime} / 2\right)} e^{-\beta x}}\left(\frac{d}{d x}\right)^{n-m^{\prime}}\left(x^{n+\alpha} e^{-\beta x}\right),
$$

where $\alpha_{n, m^{\prime}}$ is the normalization coefficient and will be obtained later. As mentioned in $[10,11]$, we can write the associated Laguerre differential equation (21) as the following shape invariant equations with respect to the parameter $m^{\prime}$ :

$$
\begin{aligned}
A_{m^{\prime}}^{+}(x) A_{m^{\prime}}^{-}(x) L_{n, m^{\prime}}^{(\alpha, \beta)}(x) & =\left(n-m^{\prime}+1\right) \beta L_{n, m^{\prime}}^{(\alpha, \beta)}(x), \\
A_{m^{\prime}}^{-}(x) A_{m^{\prime}}^{+}(x) L_{n, m^{\prime}-1}^{(\alpha, \beta)}(x) & =\left(n-m^{\prime}+1\right) \beta L_{n, m^{\prime}-1}^{(\alpha, \beta)}(x),
\end{aligned}
$$

where operators $A_{m^{\prime}}^{+}(x)$ and $A_{m^{\prime}}^{-}(x)$ are given by the following equation:

$$
\begin{gathered}
A_{m^{\prime}}^{+}(x)=\sqrt{x} \frac{d}{d x}-\frac{m^{\prime}-1}{2 \sqrt{x}}, \\
A_{m^{\prime}}^{-}(x)=-\sqrt{x} \frac{d}{d x}-\frac{2 \alpha+m^{\prime}-2 \beta x}{2 \sqrt{x}} .
\end{gathered}
$$

One may write down the shape invariance equation (23) as the raising and lowering relations:

$$
\begin{gathered}
A_{m^{\prime}}^{+}(x) L_{n, m^{\prime}}^{(\alpha, \beta)}(x)=\sqrt{\left(n-m^{\prime}+1\right) \beta} L_{n, m^{\prime}}^{(\alpha, \beta)}(x), \\
A_{m^{\prime}}^{-}(x) L_{n, m^{\prime}}^{(\alpha, \beta)}(x)=\sqrt{\left(n-m^{\prime}+1\right) \beta} L_{n, m^{\prime}-1}^{(\alpha, \beta)}(x) .
\end{gathered}
$$

On the other hand, the associated Laguerre differential equation (21) can be factorized with respect to the parameter $n$ for a given $m^{\prime}$ as

$$
\begin{aligned}
& A_{n, m^{\prime}}^{+}(x) A_{n, m^{\prime}}^{-}(x) L_{n, m^{\prime}}^{(\alpha, \beta)}(x) \\
& \quad=\left(n-m^{\prime}\right)(n+\alpha) \beta L_{n, m^{\prime}}^{(\alpha, \beta)}(x), \\
& A_{n, m^{\prime}}^{-}(x) A_{n, m^{\prime}}^{+}(x) L_{n-1, m^{\prime}}^{(\alpha, \beta)}(x) \\
& \quad=\left(n-m^{\prime}\right)(n+\alpha) \beta L_{n-1, m^{\prime}}^{(\alpha, \beta)}(x),
\end{aligned}
$$

where the differential operators are functions of parameters $n$ and $m^{\prime}$ which are obtained as

$$
\begin{gathered}
A_{n, m^{\prime}}^{+}(x)=x \frac{d}{d x}-\beta x+\frac{1}{2}\left(2 n+2 \alpha-m^{\prime}\right), \\
A_{n, m^{\prime}}^{-}(x)=-x \frac{d}{d x}+\frac{1}{2}\left(2 n-m^{\prime}\right) .
\end{gathered}
$$

Note that the shape invariant equation (28) can be written as the raising and lowering relations

$$
\begin{aligned}
& A_{n, m^{\prime}}^{+}(x) L_{n-1, m^{\prime}}^{(\alpha, \beta)}(x)=\sqrt{\left(n-m^{\prime}\right)(n+\alpha)} L_{n, m^{\prime}}^{(\alpha, \beta)}(x), \\
& A_{n, m^{\prime}}^{-}(x) L_{n, m^{\prime}}^{(\alpha, \beta)}(x)=\sqrt{\left(n-m^{\prime}\right)(n+\alpha) \beta} L_{n-1, m^{\prime}}^{(\alpha, \beta)}(x) .
\end{aligned}
$$


The above mentioned method with some calculations leads to the following normalization coefficient:

$$
\alpha_{n, m^{\prime}}(\alpha, \beta)=(-1)^{n} \sqrt{\frac{\beta^{\alpha+m^{\prime}+1}}{\Gamma\left(n-m^{\prime}+1\right) \Gamma(n+\alpha+1)}} .
$$

Also, the normalization coefficient equation (29) has been so chosen that the associated Laguerre functions $L_{n, m^{\prime}}^{(\alpha, \beta)}(x)$, with the same $m^{\prime}$ but with different $n$ with respect to the inner product with the weight functions $x^{\alpha} e^{-\beta x}$, form an orthonormal set in the interval $0 \leq x<\infty$ :

$$
\int_{0}^{\infty} L_{n, m^{\prime}}^{(\alpha, \beta)}(x) L_{n^{\prime}, m^{\prime}}^{(\alpha, \beta)}(x) x^{\alpha} e^{-\beta x} d x=\delta_{n, n^{\prime}} .
$$

Now, we go back to the Dirac oscillator with AharonovCasher system and compare it with associated Laguerre equation.

\section{Factorization Method and Dirac Oscillator with Aharonov-Casher System}

We wish to get a solution for (18) which will be regular at the origin; then we have to compare (20) to (21) and obtain $F(x)$ as

$$
F(x)=x^{\alpha / 2} e^{-(\beta / 2) x},
$$

where $x=m \omega \rho^{2}$. Comparing equation (20) and (21), yields to the following conditions:

$$
\begin{aligned}
\beta & = \pm \frac{1}{2} \\
\xi_{s} & =m^{\prime}+\alpha \\
B_{s} & =4 m \omega\left(n-\frac{m^{\prime}}{2}\right)+2 m \omega(\alpha+1) .
\end{aligned}
$$

The associated Laguerre polynomial and regular solution at the origin lead us to take $\beta=1 / 2$. Equation (32) helps us to obtain the energy spectrum for the Dirac oscillator with Aharonov-Casher system. In order to specify the energy spectrum, we consider two cases. First, for obtaining the positive-energy solution of Dirac equation $(\Psi)$, we consider the component parallel to $z$-axis of the space-time; we must take $s=+1$ and consider $\varphi_{-}=0$. The energy spectrum is

$$
\varepsilon^{2}=4 m \omega\left(n-m^{\prime}\right)+m^{2}+k^{2} .
$$

We see the energy is positive because always we have $n \geq m^{\prime}>$ 0 . In second case, we consider the positive-energy solutions corresponding to the $s=-1$ and $\varphi_{+}=0$. So, we can obtain,

$$
\varepsilon^{2}=4 m \omega(n+\alpha)+m^{2}+k^{2} .
$$

We can see in two cases the energy compound to AharonovCasher effect in the Minkowski space-time. So, the general wave function with $x=m \omega \rho^{2}$ and condition (32) will be as

$$
\begin{aligned}
\phi_{s}(x) & =A e^{i(\ell+(1 / 2)) \varphi} e^{i k z} F(x) L_{n, m^{\prime}}^{(\alpha,(1 / 2))}(x) \\
& =A e^{i(\ell+(1 / 2)) \varphi} e^{i k z}(m \omega)^{\alpha / 2} x^{\alpha} e^{-(1 / 4) m \omega \rho^{2}} L_{n, m^{\prime}}^{(\alpha, 1 / 2)}(x), \\
\phi_{s}(\varphi) & =A(m \omega)^{\alpha / 2} e^{i(\ell+(1 / 2)) \varphi} e^{i k z} \rho^{\alpha} e^{-(1 / 4) m \omega \rho^{2}} L_{n, \xi_{s}-\alpha}^{(\alpha,(1 / 2))}(\rho) .
\end{aligned}
$$

So, the associated Laguerre function obtained as a solution of the differential (20) has the following Rodrigues representation:

$$
\begin{aligned}
& L_{n, \xi_{s}-\alpha}(\rho) \\
& \quad=\frac{\alpha_{n, \xi_{s}-\alpha}(\alpha, 1 / 2)}{\rho^{\alpha+\xi_{s}} e^{-\left(m \omega \rho^{2} / 2\right)}}\left(\frac{1}{\rho} \frac{d}{d \rho}\right)^{n-\xi_{s}+\alpha}\left(\rho^{2 n+2 \alpha} e^{-m \omega \rho^{2} / 2}\right),
\end{aligned}
$$

where $\alpha_{n, \xi_{s}-\alpha}(\alpha, 1 / 2)$ will be as

$$
\begin{aligned}
& \alpha_{n, \xi_{s}-\alpha}\left(\alpha, \frac{1}{2}\right) \\
& =(-1)^{n} \sqrt{\frac{2^{3 \xi_{s}-2 n-2 \alpha}}{\Gamma\left(n-\xi_{s}+\alpha\right) \Gamma(n+\alpha+1)(m \omega)^{\alpha-\xi_{s}}}} .
\end{aligned}
$$

As mentioned in $[10,11]$, we can write the associated Laguerre differential equation (20) as the following shape invariant equations with respect to the parameter $\xi_{s}-\alpha=m^{\prime}$ :

$$
\begin{aligned}
& A_{\xi_{s}-\alpha}^{+}(\rho) A_{\xi_{s}-\alpha}^{-}(\rho) L_{n, \xi_{s}-\alpha}^{(\alpha, 1 / 2)}(\rho) \\
& =\left(n-\xi_{s}+\alpha+1\right) \frac{1}{2} L_{n, \xi_{s}-\alpha}^{(\alpha, 1 / 2)}(\rho), \\
& A_{\xi_{s}-\alpha}^{-}(\rho) A_{\xi_{s}-\alpha}^{+}(\rho) L_{n, \xi_{s}-\alpha-1}^{(\alpha, 1 / 2)}(\rho) \\
& =\left(n-\xi_{s}+\alpha+1\right) \frac{1}{2} L_{n, \xi_{s}-\alpha-1}^{(\alpha, 1 / 2)}(\rho),
\end{aligned}
$$

where operators $A_{\xi_{s}-\alpha}^{+}(\rho)$ and $A_{\xi_{s}-\alpha}^{-}(\rho)$ are given by

$$
\begin{gathered}
A_{\xi_{s}-\alpha}^{+}(\rho)=\frac{1}{2 \sqrt{m \omega}}\left(\frac{d}{d \rho}-\frac{\xi_{s}-\alpha-1}{\rho}\right), \\
A_{\xi_{s}-\alpha}^{-}(x)=-\frac{1}{2 \sqrt{m \omega}}\left(\frac{d}{d \rho}+\frac{\alpha+\xi_{s}-m \omega \rho^{2}}{\rho}\right) .
\end{gathered}
$$

One may write down the shape invariance equation (1) as the raising and lowering relations

$$
\begin{gathered}
A_{\xi_{s}-\alpha}^{+}(\rho) L_{n, \xi_{s}-\alpha}^{(\alpha, 1 / 2)}(\rho)=\sqrt{\left(n-\xi_{s}+\alpha+1\right) \frac{1}{2}} L_{n, \xi_{s}-\alpha}^{(\alpha, 1 / 2)}(\rho), \\
A_{\xi_{s}-\alpha}^{-}(\rho) L_{n, \xi_{s}-\alpha}^{(\alpha, 1 / 2)}(\rho)=\sqrt{\left(n-\xi_{s}+\alpha+1\right) \frac{1}{2}} L_{n, \xi_{s}-\alpha-1}^{(\alpha, 1 / 2)}(\rho) .
\end{gathered}
$$


On the other hand, the associated Laguerre differential equation (20) can be factorized with respect to the parameter $n$ for a given $n$ as

$$
\begin{aligned}
& A_{n, \xi_{s}-\alpha}^{+}(\rho) A_{n, \xi_{s}-\alpha}^{-}(\rho) L_{n, \xi_{s}-\alpha}^{(\alpha, 1 / 2)}(\rho) \\
& =\frac{1}{2}\left(n-\xi_{s}+\alpha\right)(n+\alpha) L_{n, \xi_{s}-\alpha}^{(\alpha, 1 / 2)}(\rho), \\
& A_{n, \xi_{s}-\alpha}^{-}(\rho) A_{n, \xi_{s}-\alpha}^{+}(\rho) L_{n-1, \xi_{s}-\alpha}^{(\alpha, 1 / 2)}(\rho) \\
& =\frac{1}{2}\left(n-\xi_{s}+\alpha\right)(n+\alpha) L_{n-1, \xi_{s}-\alpha}^{(\alpha, 1 / 2)}(\rho),
\end{aligned}
$$

where the differential operators are functions of parameters $n$ and $\xi_{s}-\alpha$ which are obtained by

$$
\begin{gathered}
A_{n, \xi_{s}-\alpha}^{+}(\rho)=\frac{1}{2} \rho \frac{d}{d \rho}-\frac{1}{2} m \omega \rho^{2}+\frac{1}{2}\left(2 n+3 \alpha-\xi_{s}\right), \\
A_{n, \xi_{s}-\alpha}^{-}(\rho)=-\frac{1}{2} \rho \frac{d}{d \rho}+\frac{1}{2}\left(2 n-\xi_{s}+\alpha\right) .
\end{gathered}
$$

Note that the shape invariant equation (27) can be written as the raising and lowering relations

$$
\begin{aligned}
& A_{n, \xi_{s}-\alpha}^{+}(\rho) L_{n-1, \xi_{s}-\alpha}^{(\alpha, 1 / 2)}(\rho)=\sqrt{\left(n-\xi_{s}+\alpha\right)(n+\alpha)} L_{n, \xi_{s}-\alpha}^{(\alpha, 1 / 2)}(\rho), \\
& A_{n, \xi_{s}-\alpha}^{-}(\rho) L_{n, \xi_{s}-\alpha}^{(\alpha, 1 / 2)}(\rho)=\sqrt{\left(n-\xi_{s}+\alpha\right)(n+\alpha)} L_{n-1, \xi_{s}-\alpha}^{(\alpha, 1 / 2)}(\rho) .
\end{aligned}
$$

Here, we note that the factorization method and shape invariance condition help us to factorize the second order oscillator with the Aharonov-Casher equation. It means that such equation factorized in terms of two first order operators which are known by raising and lowering operators.

\section{Conclusion}

In this paper, first we introduced the nonlinear equation corresponding with oscillator and Aharonov-Casher system. We could easily solve such system by using factorization method and shape invariance condition. Also, the factorization method helps us to obtain the general form of wave function and energy spectrum which is hard to obtain with ordinary methods. Finally, we achieved the first order differential equation as raising and lowering operators. It may be interesting to show that such operators can be a form of generators of $N=2$ supersymmetry algebras. Another problem for such system is partner Hamiltonian with new and modified potential. We can do such complicated problem in future.

\section{Conflict of Interests}

The authors declare that there is no conflict of interests regarding the publication of this paper.

\section{References}

[1] Y. Aharonov and A. Casher, "Topological quantum effects for neutral particles," Physical Review Letters, vol. 53, no. 4, pp. 319321, 1984.

[2] J. Anandan, "Electromagnetic effects in the quantum interference of dipoles," Physics Letters A, vol. 138, no. 8, pp. 374-352, 1989.

[3] C. Brans and J. Anandan, "Classical and quantum interaction of the dipole," Physical Review Letters, vol. 85, p. 1354, 2000.

[4] W. Greiner, Relativistic Quantum Mechanics: Wave Equations, Springer, Berlin, Germany, 3rd edition, 2000.

[5] N. D. Birrell and P. C. W. Davies, Quantum Fields in Curved Space, vol. 7 of Cambridge Monographs on Mathematical Physics, Cambridge University Press, Cambridge, Mass, USA, 1982.

[6] M. Nakahara, Geometry, Topology and Physics, Institute of Physics, Bristol, UK, 1998.

[7] S.-H. Dong, Factorization Method in Quantum Mechanics, vol. 150 of Fundamental Theories of Physics, Springer, Dordrecht, The Netherlands, 2007.

[8] M. A. Jafarizadeh and H. Fakhri, "Supersymmetry and shape invariance in differential equations of mathematical physics," Physics Letters A, vol. 230, no. 3-4, pp. 164-170, 1997.

[9] M. A. Jafarizadeh and H. Fakhri, "Parasupersymmetry and shape invariance in differential equations of mathematical physics and quantum mechanics," Annals of Physics, vol. 262, no. 2, pp. 260-276, 1998.

[10] J. Sadeghi and M. Rostami, "The shape invariance in $S^{2}$ space with electromagnetic field," International Journal of Theoretical Physics, vol. 51, no. 7, pp. 2152-2159, 2012.

[11] J. Sadeghi and B. Pourhassan, "Exact solution of the non-central modified Kratzer potential plus a ring-shaped like potential by the factorization method," Electronic Journal of Theoretical Physics, vol. 5, no. 17, pp. 197-206, 2008. 


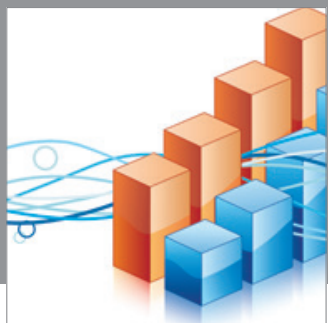

Advances in

Operations Research

mansans

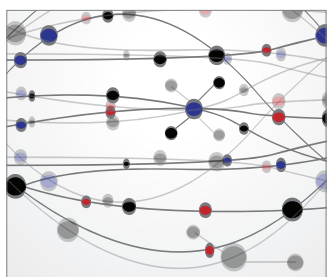

The Scientific World Journal
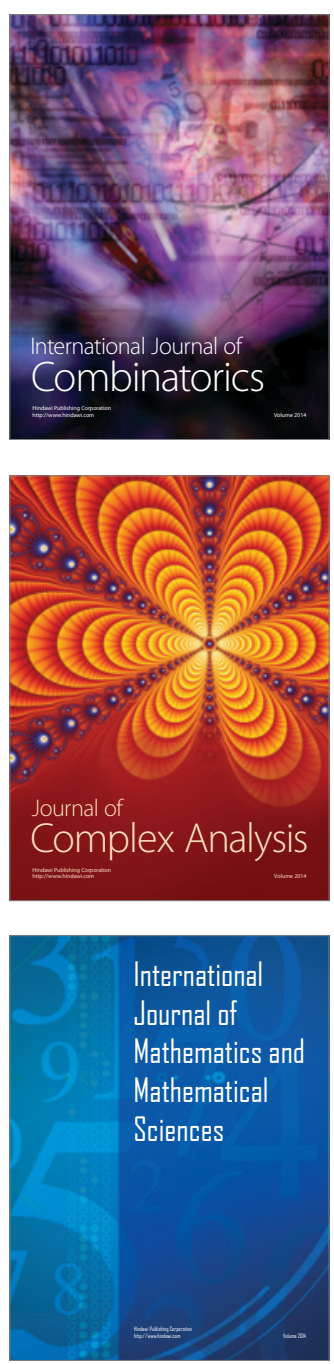
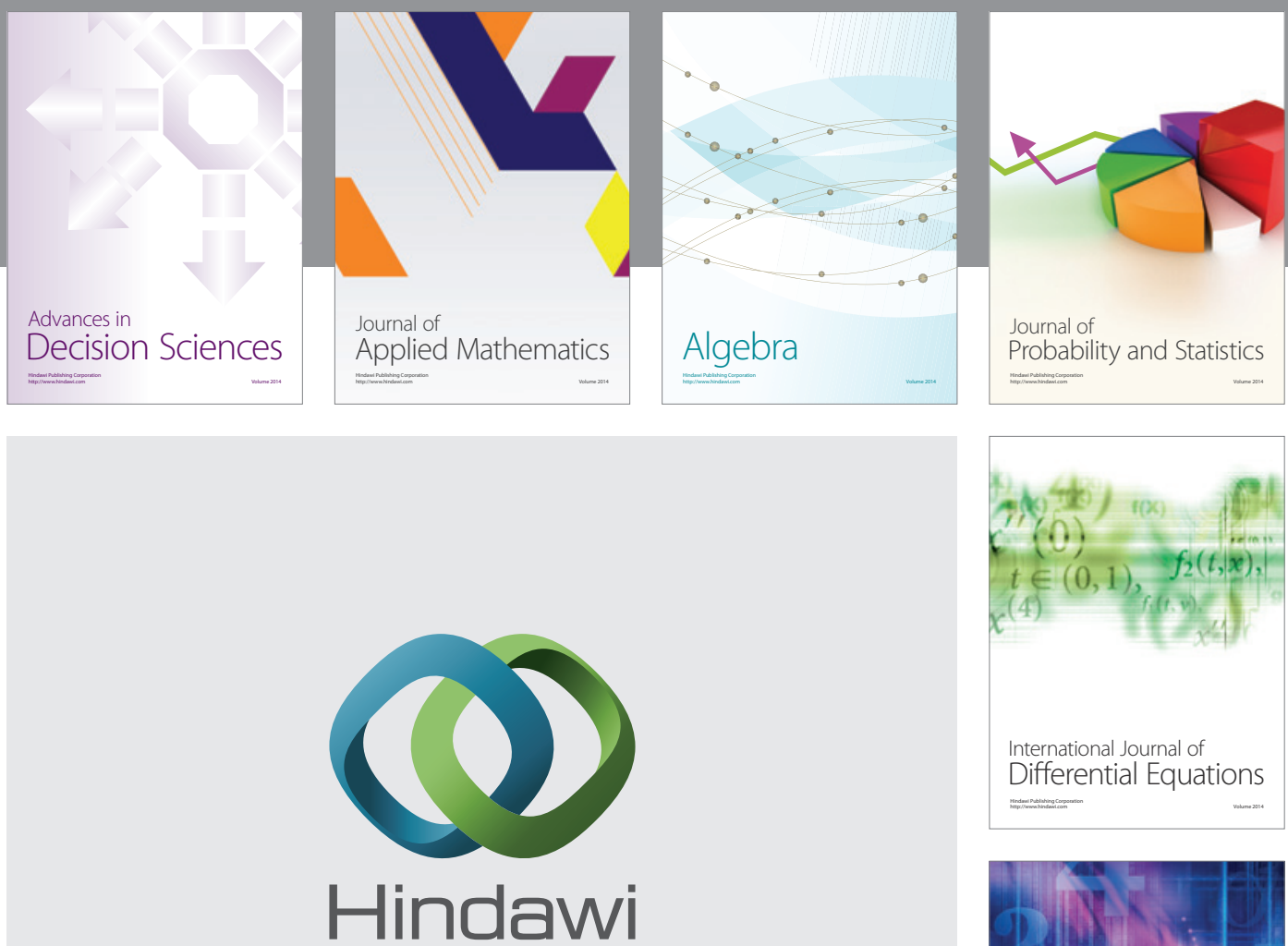

Submit your manuscripts at http://www.hindawi.com
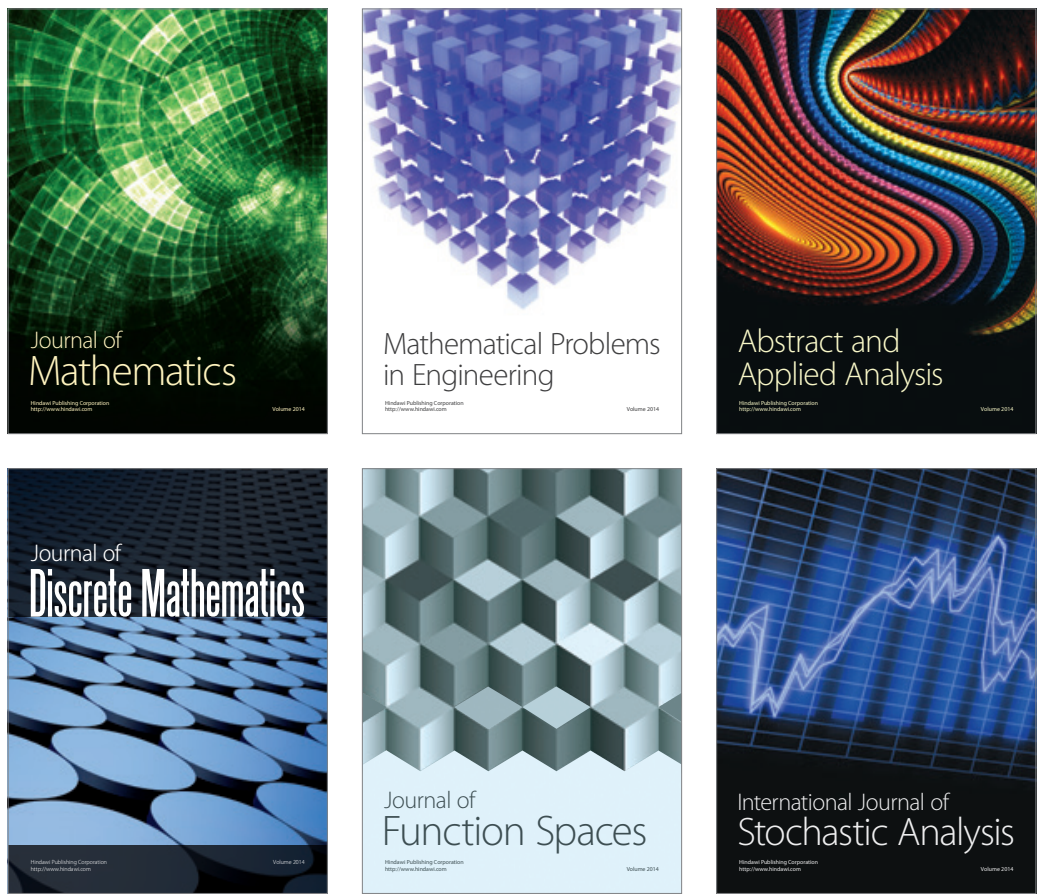

Journal of

Function Spaces

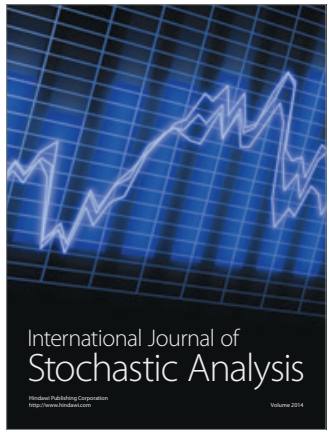

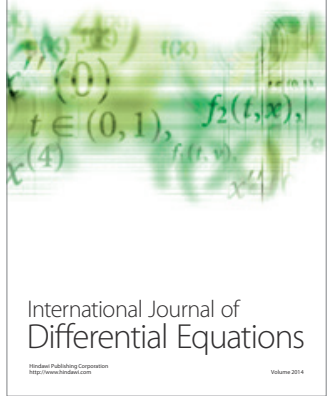
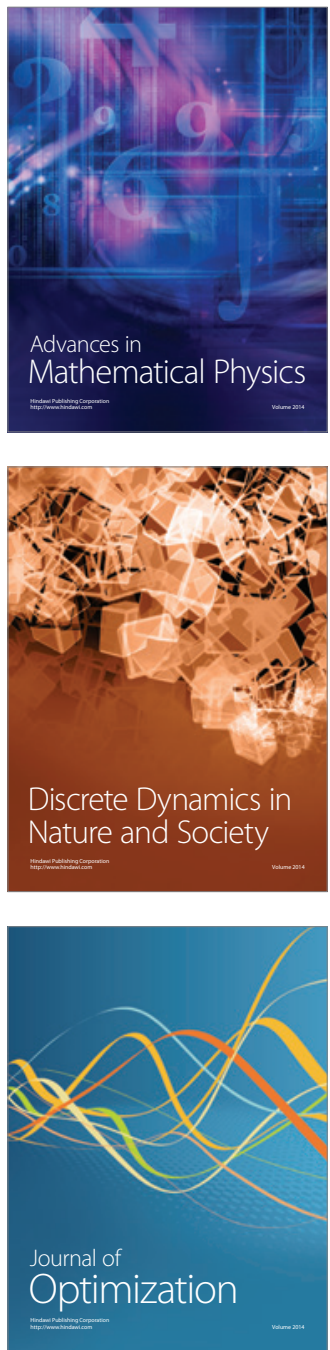